\title{
INTERFERENCE MANAGEMENT IN LTE DOWNLINK NETWORKS
}

\author{
Farhana Afroz ${ }^{1}$, Kumbesan Sandrasegaran ${ }^{2}$, H. Al Kim ${ }^{3}$ \\ Faculty of Engineering and Information Technology, \\ University of Technology, Sydney, Australia
}

\begin{abstract}
Two major challenges for evolving LTE (Long Term Evolution) networks are to achieve enhanced system capacity and cell coverage compared with WCDMA (Wideband Code Division Multiple Access) system. Effective utilization of radio resources as well as dense spectrum reuse are at the core to attain these targets. However, dense frequency reuse may increase inter-cell interference, which in turn severely limits the capacity of users in the system. Inter-cell interference can restrict overall system performance in terms of throughput and spectral efficiency, especially for the users located at the cell edge area. Hence, careful management of inter-cell interferences becomes crucial to improve LTE system performance. In this paper, interference mitigation schemes for LTE downlink networks are investigated.
\end{abstract}

\section{KEYWORDS}

LTE, OFDMA, interference avoidance, interference randomization, resource block, SINR, throughput

\section{INTRODUCTION}

The growing demand of providing ubiquitous broadband internet access on mobile networks has imposed the need of developing OFDMA (Orthogonal Frequency Division Multiple Access) based wireless cellular networks such as $4 \mathrm{G}$ networks. One of the major challenges for evolving LTE networks is to increase network capacity [1]. Though dense frequency reuse results in significant system capacity improvement, it also remarkably degrades the performance of the system due to the increase in interference caused by adjacent cells [1,2]. In cellular mobile communication system, mainly two types of interference must be taken into consideration such as intra-cell interference and inter-cell interference. In intra-cell interference (shown in Fig. 1(a)), interfering mobile terminal is in the same cell. The spillover transmission between adjacent channels within a cell results in intra-cell interference. In intercell interference (ICI) (shown in Fig. 1(b)), interfering mobile terminal is in adjacent cell. ICI is caused by the use of the same frequency channel in neighbouring cells [3].

In LTE downlink (DL), OFDMA radio access technology is used where the subcarriers are mutually orthogonal to each other, implying that there is no intra-cell interference. However, ICI can limit system performance in terms of throughput and spectral efficiency, especially for users located at the cell edge. So, careful management of inter-cell interference is very important in LTE to improve system performance [3,4].

To mitigate inter-cell interference (ICI), some strategies are used during the transmission or after the reception of the signal. ICI mitigation techniques can be classified as interference 
randomization, interference cancellation and interference avoidance. In the first strategy, some cell specific scrambling, interleaving or spread spectrum techniques can be used to reduce interference. The

interference is distributed randomly among all users such as using pseudorandom scrambling after channel coding. Thus, the cell edge users will not always suffer strong ICI during the entire transmission period. In interference cancellation, the interfering signal is regenerated through signal processing and the estimated interfering signal is subtracted from the received signal (desired signal + interference) [5].

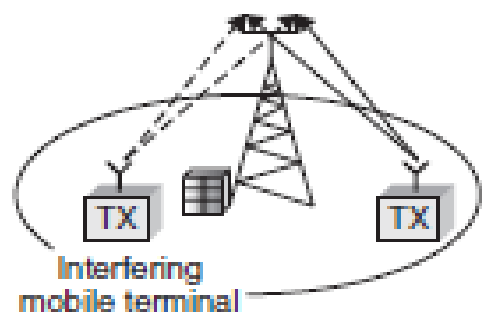

[a]

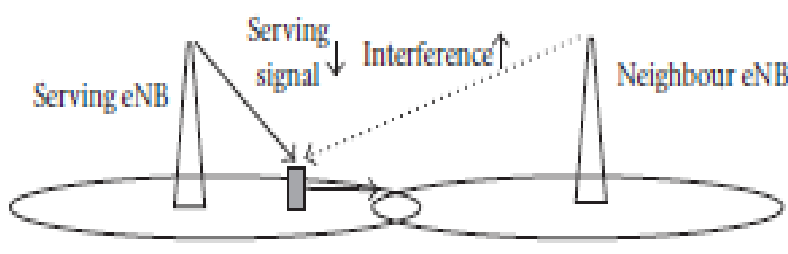

[b]

Fig. 1: (a) Intra-cell interference [3] (b) Inter-cell interference [12]

In interference avoidance, the allocation of various resources (e.g. time/frequency/power) is controlled to increase signal to interference and noise ratio, SINR; thereby throughput, for cell edge users and to make sure ICI will be within tolerable limits [6]. Interference avoidance strategy ensures better services for users located at cell edge without sacrificing cell centre users' throughput.

The benefits of using each of these ICI mitigation strategies mentioned above are mutually exclusive. Hence, combination of these schemes is anticipated in imminent systems. Effective utilization of radio resources such as power control, coordinated packet scheduling plays very significant role in mitigating interference. This paper does not cover power control techniques. In this paper, we mainly focus on interference avoidance schemes which are more related to the DL (Downlink) LTE networks. A short overview of interference randomization is also provided.

The rest of the paper is organized as follows. An overview of inter-cell interference is provided in section 2. Section 3 summarizes existing interference avoidance schemes followed by fundamentals of interference randomization in section 4 . Finally, section 5 concludes the paper. 


\section{INTER-CELL INTERFERENCE (ICI)}

In LTE, the smallest unit of radio resource that can be allocated to a user for data transmission during packet scheduling is called physical resource block (PRB). Radio resources are defined in time-frequency domain (shown in upper part of Fig. 2). A time/frequency radio resource that spans over one time slot of $0.5 \mathrm{~ms}$ in the time domain and one sub-channel $(180 \mathrm{KHz})$ of 12 subcarriers in the

frequency domain is known as resource block (RB). The RB pairs (in time domain) are allocated to a UE for data transmission in a TTI (Transmission Time Interval= $1 \mathrm{~ms}$ ). Inter-cell interference is caused as a result of collisions between RBs that are utilized by multiple cells simultaneously $[6,7]$. The PRBs reusing by UEs located at adjacent cells result in ICI in OFDMA systems [8]. When a user moves away from serving eNB and becomes closer to its adjacent eNB, the received SINR (Signal to Interference and Noise Ratio) degrades as the desired received signal power decreases and the ICI increases. The impact of inter-cell interference in LTE DL can be analysed by calculating the received SINR of $\mathrm{UE}_{m}$ on the $\mathrm{RB}_{\mathrm{n}}$ (Resource Block n) according to the equation (1) [2].
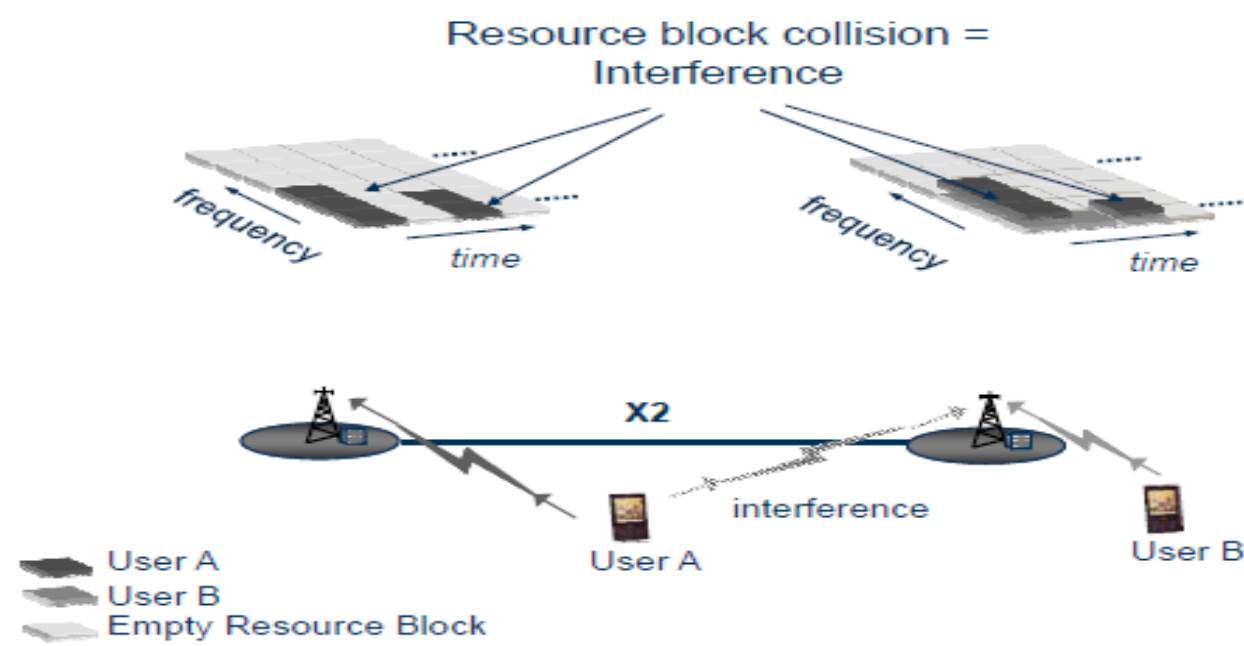

Fig. 2: Fundamentals of inter-cell interference in OFMDA systems [7]

$$
S I N R_{m, n}=\frac{p_{n}^{l} H_{m, n}^{l}}{{ }_{k \neq l} p_{n}^{k} H_{m, n}^{k} \delta_{n}^{k}+P_{N}}
$$

Where, $\operatorname{SINR}_{m, n}$ is the SINR of UE $m$ on RB $n$

$p_{n}^{l}$ is the transmission power from the serving cell $l$ on $\mathrm{RB} n$

$H_{m, n}^{k}$ is the channel gain from the cell $l$ to $\mathrm{UE} m$ on $\mathrm{RB} n$

$P_{N}$ is the noise power

$\delta_{n}^{k}$ is an indicator which is set to 1 or 0 to specify whether the neighbouring cell $k$ allocates $\mathrm{RB} n$ to its UEs or not.

From equation (1), it is clear that there are three significant factors named channel gain, transmit power and RB allocation scheme which have a great impact on SINR of each UE. SINR can be decreased in three situations such as when UE is far away from serving eNB which in turn decreases channel gain, when transmit power of adjacent cell on a RB increases 
which thereby increases interference, and when adjacent cells assign the same RB to their UEs. Thus, the SINRs of cell centre users (CCU) located nearer to their serving eNB are better as compared with the users located at the edges.

A range of power and frequency allocation strategies can be adopted for cell centre and cell edge users to mitigate ICI. Though an increase in transmit power can improve the signal to interference and noise ratio, it may significantly increase the overall interference of the system. Thus for each UE, particularly cell edge user (CEU), an increase in user's transmit power imposes a conflict over the

overall system performance. So, various power allocation schemes are proposed to obtain a trade-off between the achieved SINR and resulting interference so as the system's performance can be improved [9]. Considering the third situation when ICI increases (when the neighbor cells allocate the same RB to their users), various RB allocation schemes are adopted as ICI mitigation schemes in which the objective of the schemes is to reduce ICI and maintain the higher spectral efficiency concurrently. It is also observed from the equation (1) that if the serving cell selects $\mathrm{RB}_{\mathrm{n}}$ to transmit data, the ICI can be reduced noticeably if the adjacent cells do not assign the same $\mathrm{RB}_{\mathrm{n}}$ to their users i.e. when $\delta_{n}^{k}=0$. The allocation granularity can be a resource block or a part of available bandwidth. The conception of reserving specific portions of the bandwidth for CCU and CEU to avoid interference is named as frequency reuse technique.

\section{INTERFERENCE AvoIDANCE SCHEMES}

In cellular network, different power and frequency allocation schemes are deployed to avoid the impact of ICI so as the system's spectral efficiency can be improved. In many ICI mitigation schemes, frequency reuse technique is taken as the main idea. These frequency reuse planning algorithms aim to improve the SINR, and must fulfil the power constraint of each cell by making sure that the transmit power of an eNB is not exceeding the maximum allowable limit. Based on time scale, ICI avoidance schemes can be categorized as static, semi-static and dynamic schemes. Static allocation schemes can operate on a relatively large time scale $[10,11]$. In static scheme, the resource allocation for each cell is determined during radio planning and only long-term readjustments are made during network operation. Therefore, the power levels and the set of sub-carriers assigned for each cell and cell regions are static (fixed). In semi-static approach, a part of the RB allocation is predefined and the other RBs allocations reserved for cell edge users are dynamically changed. Timescale of reallocation is in seconds or several hundred milliseconds. In dynamic scheme, resource allocation is dynamically updated based on the variations of network conditions. Dynamic allocations are done after a very short time period [2, 11]. In the following subsections, frequency reuse schemes are classified into static, semi-static and dynamic schemes.

\subsection{Static Schemes}

In spite of distinct differences between different frequency reuse schemes, all schemes need to specify the set of sub-carriers allocated for each cell/sector, the power level at which each channel operates, and the cell regions (i.e. cell centre or cell edge) in which the set of subcarriers (channels) are utilized. Various frequency reuse schemes specify different values for these parameters [6].

\subsubsection{Conventional Frequency Reuse}

The simplest frequency reuse scheme is to utilize a frequency reuse factor one (RF1). In RF1, the total available bandwidth is reused in each cell without posing any constraints on power 
allocation or frequency resource usage (as shown in Fig. 3(a)). With this scheme, high system capacity i.e. high peak data rate can be achieved. However, inter-cell interference, especially at cell edges, is increased which in turn considerably limits the performance of cell-edge users. Thus, the overall spectral efficiency degrades.

In reuse factor three (RF3), the total bandwidth is divided into three equal and orthogonal subbands and the sub-bands are allocated to cells in such a way that adjacent cells always deploy different frequencies (shown in Fig. 3(b)). This scheme leads to lower inter-cell interference. However, as each cell is using one third of total available bandwidth, there is a large capacity loss.
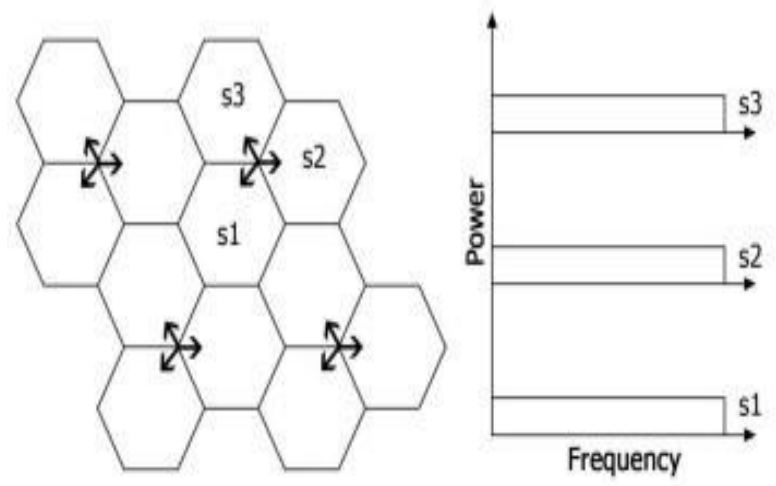

(a)

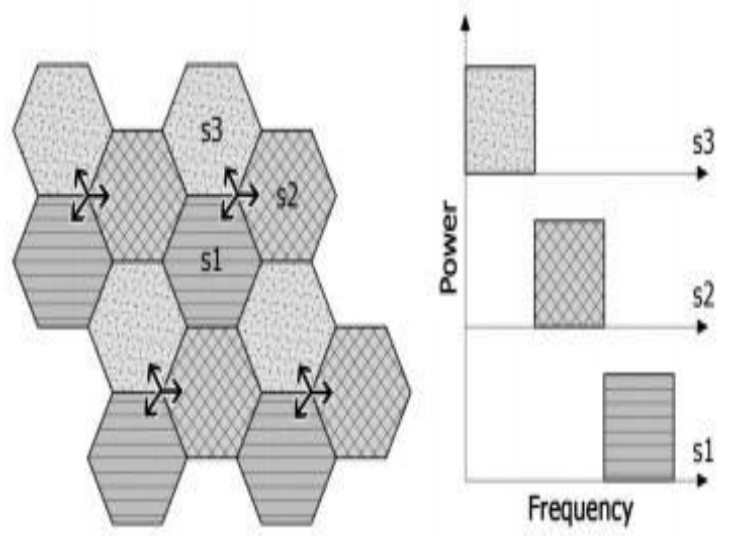

(b)

Fig. 3: Conventional frequency planning (a) frequency reuse factor 1 (b) requency reuse factor 3 [6]

\subsubsection{Fractional Frequency Reuse (FFR)}

Fractional frequency reuse scheme was proposed to overcome the shortcomings of conventional frequency reuse schemes. In FFR- based schemes, the users with a stronger signal quality use a lower reuse factor scheme (such as RF1) and the users having lower SINR use a higher reuse factor schemes (such as RF3) [2].

\subsubsection{Partial Frequency Reuse (PFR)}

It is not bandwidth efficient to reuse the same frequency reuse factor (FRF) in the entire cell [12]. A solution to increase the SINR of cell edge users, while good spectral efficiency is being maintained, is to use a reuse factor greater than one for cell edge regions and RF1 for 
cell centre regions [13]. The basic concept of PFR is to put restrictions on a portion of the resources so as some resources are not utilized by some user classes at all. In PFR scheme (shown in Fig. 4), total available bandwidth is divided into four sub-bands. Cell centre UEs are allocated in the frequency band using reuse factor of 1 . Cell edge UEs are allocated in the complementary frequency band using reuse factor of 3. This scheme is also known as FFR-FI (FFR with full isolation) as the cell edge users are completely isolated. As PFR does not employ the whole available bandwidth, it leads to lower cell throughput compared with RF1 scheme.

\subsubsection{Soft Frequency Reuse (SFR)}

One shortcoming of PFR scheme is - it may under-utilize the available frequency resources because of its restricted no-sharing policy. Soft frequency reuse scheme proposed in $[14,15]$, was aimed to avoid the high inter-cell interference associated with reuse factor 1 configuration while more flexibility is being provided to the PFR scheme. In SFR (shown in Fig. 5), each cell uses the total available bandwidth. For each sector, cell edge users are allocated in the fraction of bandwidth with highest power level and cell centre users are allocated with lower power in the rest of the frequency band. RF1 is used in the cell centre region and FRF greater than one is employed at the cell edge regions.

An enhancement of SFR scheme is known as SFFR (Soft Fractional Frequency Reuse). The SFR and PFR techniques can enhance the throughput of the users at the cell edge region by minimizing the inter-cell interference experienced by cell-edge users. However, these schemes may result in lower cell throughput compared with conventional RF1 scheme. As PFR does not employ the whole bandwidth available in the cell, it leads to lower cell throughput compared with RF1 scheme. Furthermore, though SFR may improve overall system capacity as compared with PFR (as SFR can utilize the whole available bandwidth), however SFR may lead to lower overall system capacity than RF1 scheme. SFFR scheme was proposed to improve the overall cell throughput. In soft FFR, the resources allocated to the users at the cell edges of other cell are allowed to be used by the other cells users (inner) with some power restriction (as shown in Fig. 6) [16].

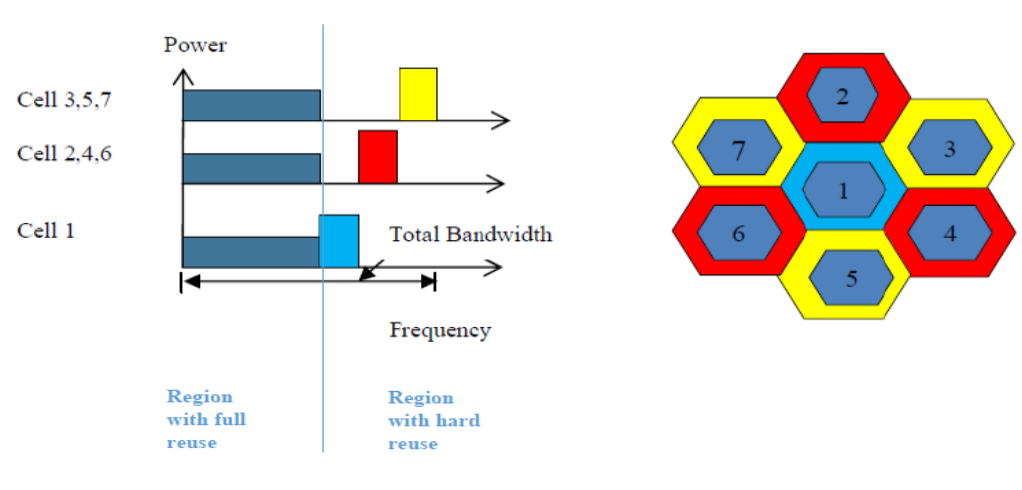

Fig. 4: Frequency planning and power allocation for PFR scheme 
International Journal of Wireless \& Mobile Networks (IJWMN) Vol. 7, No. 1, February 2015
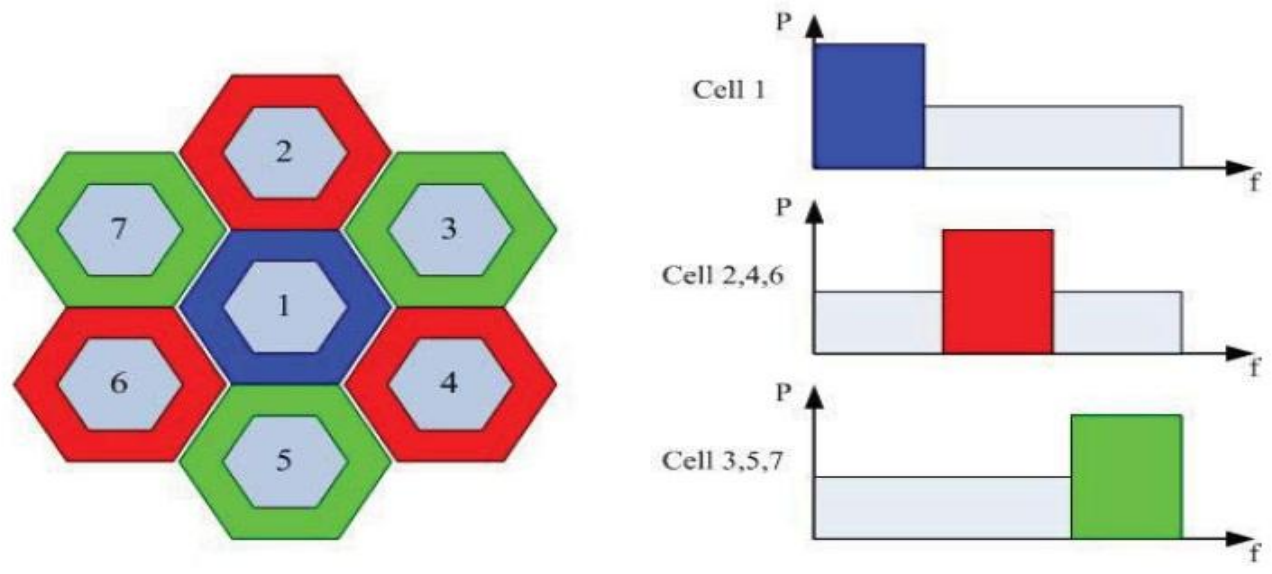

Fig. 5: Frequency planning and power allocation for SFR scheme
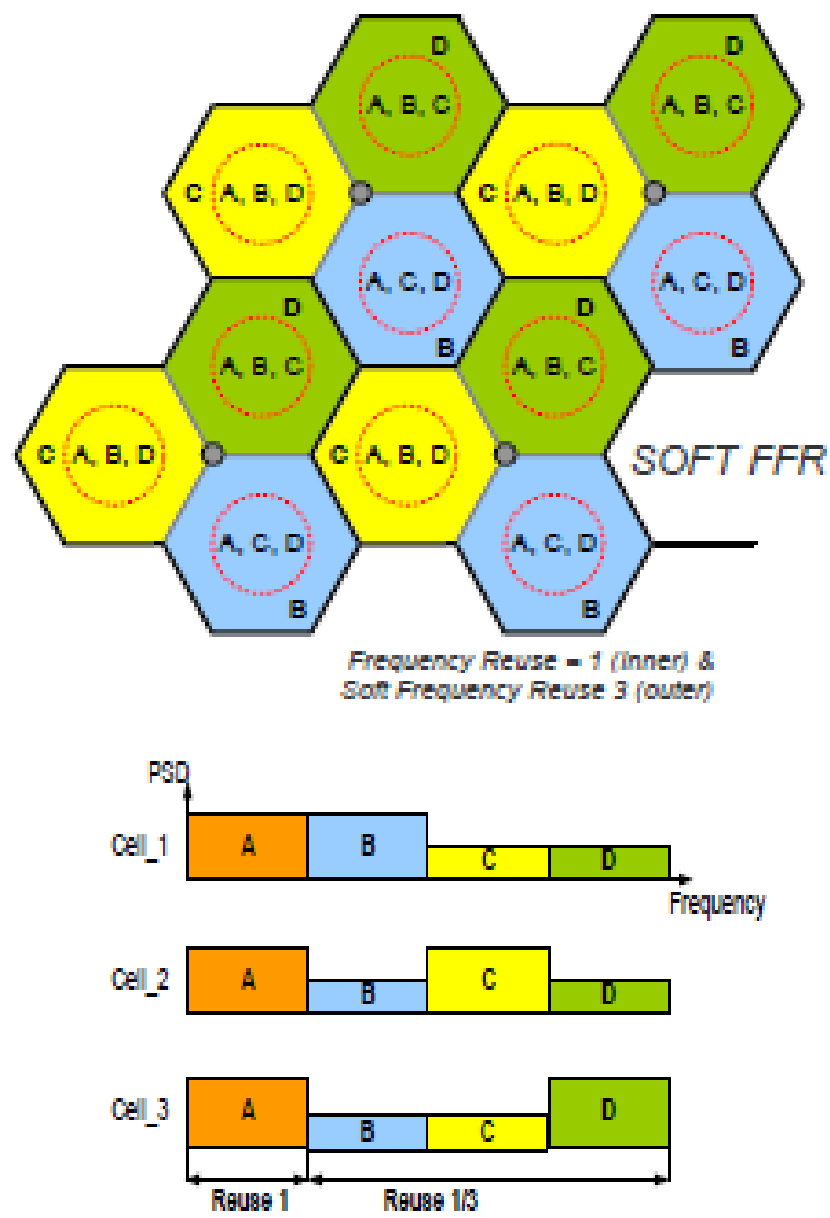

Fig.6: Frequency planning and power allocation for SFFR scheme [16] 

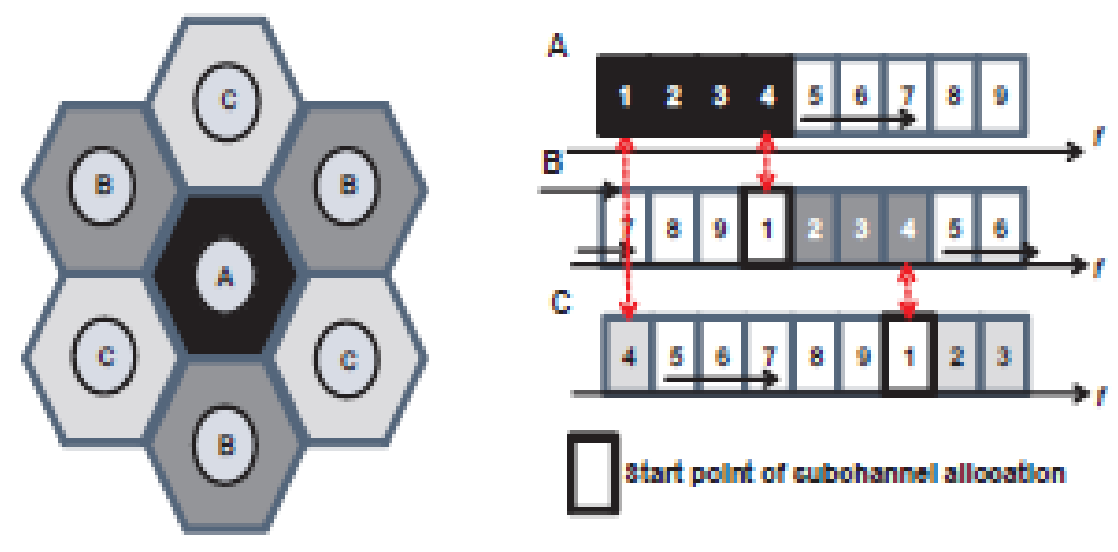

Fig. 7: Incremental Frequency Reuse (IFR) scheme [6]
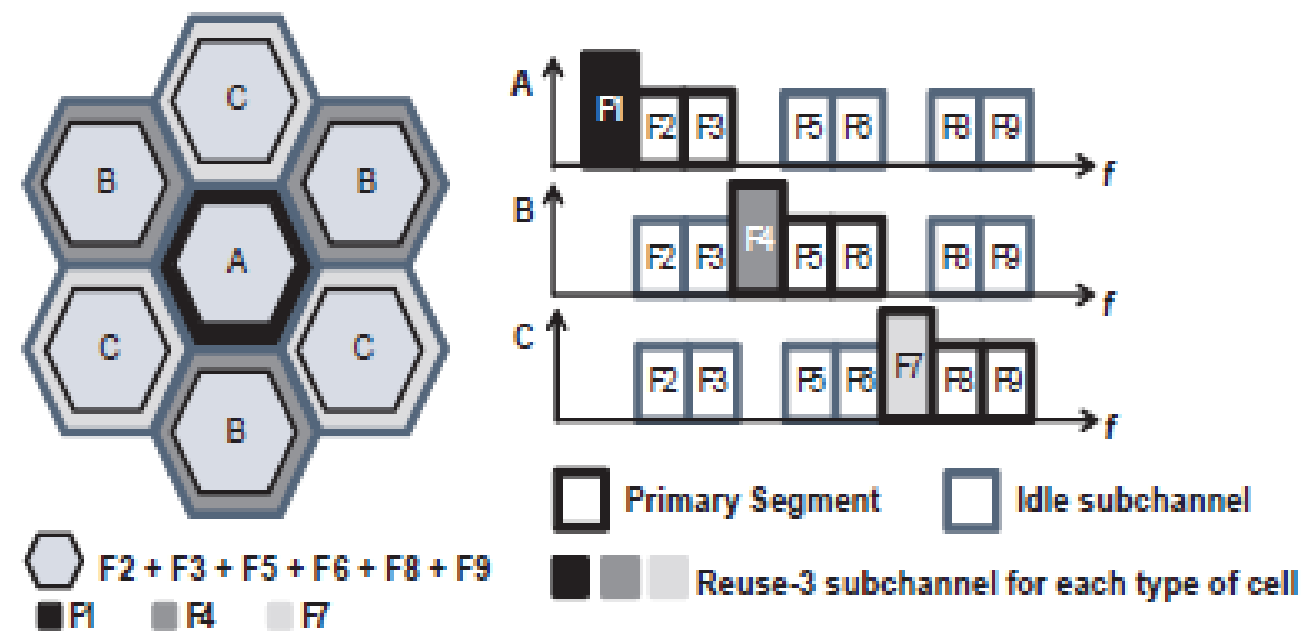

Fig. 8: Enhanced Fractional Frequency Reuse (EFFR) scheme [6]

\subsubsection{Intelligent Reuse Scheme}

In intelligent reuse scheme, frequency band assigned to different sectors expands depending on existing workloads. At low workload, this scheme starts with a RF3 like configuration and then reuse factor can be modified with the increment of workloads for becoming PFR, SFR or RF1.

\subsubsection{Incremental Frequency Reuse (IFR)}

IFR scheme was proposed to overcome some limitations of conventional SFR scheme such as low spectrum efficiency (due to the fact that the cell edge users can use maximum of $1 / 3$ of the whole bandwidth), increased co-channel interference even at low traffic load situation, overall cell capacity loss when the system is above half-full loaded. IFR aims to effectively minimize the ICI under low traffic, while overall system capacity is being maintained simultaneously. The main dissimilarity between IFR and RF1 is at which point of the available bandwidth IFR scheme starts resource allocation to the UEs. In IFR scheme, resource allocation of adjacent cells starts from different sub-channels. As shown in Fig. 7, resource allocation of type-A cells starts up from first sub-channel, whereas type-B cells from $1 / 3$ of the total available bandwidth, and type-C cell from $2 / 3$ of the whole bandwidth. Although most of the 
shortcomings of SFR scheme can be overcome by IFR scheme, it only provides better performance under low traffic situation.

\subsubsection{Enhanced Fractional Frequency Reuse (EFFR)}

EEFR was proposed to further improve the IFR and SFR system performance. EFFR aims to increase the system capacity particularly in overloaded traffic situation. As shown in Fig. 8, like IFR scheme, EFFR scheme specifies three cell-types for directly adjoining cells, and for each cell-type a portion of the total band namely Primary Segment are reserved. The Primary Segments should be orthogonal. The rest sub-channels apart from the Primary Segment form Secondary Segment. At the same time, the Primary Segment of a cell-type is a part of the Secondary Segments of other 2 cell-types. All sub-channels in each cell's Primary Segment can be occupied by this cell at will, however only a portion of sub-channels in the Secondary Segment can be occupied by this cell in inter-cell interference-aware manner. Each cell's Primary Segment is further divided into a RF3 (reuse-3) part and RF1 (reuse-1) part. The RF1 part can be reused by all cell-types, whereas RF3 part can be reused by other same cell-type. The RF3 sub-channels are forbidden to be reused by directly adjoining cells, which in turn reduces the co-channel interferences [17].

\subsection{Semi-static Schemes}

Some examples of semi-static ICI avoidance schemes are included in the following subsections.

\subsubsection{Siemen's Proposal}

As shown if Fig. 9, in frequency reuse scheme of Siemen's proposal [18, 19], the total available bandwidth is divided into $\mathrm{N}$ sub-bands. Then $\mathrm{X}$ sub-bands are utilized by the users at the cell-edges such as $X \subseteq N$. And the $\mathrm{N}-3 \mathrm{X}$ sub-bands are utilized by the cell-centre users. The X sub-bands employed for the users at the cell-edges of adjacent cells are orthogonal to each other, whereas the N-3X sub-bands employed for the users at the cell-centres are identical in all cells. The sub-band utilization of users at the cell-edges can be adopted based on the traffic load. If one more sub-band is employed for the users at the cell-edge, the subband employed for cell-centre UEs will be fall by 3 sub-bands.

\subsubsection{A Frequency Reuse Scheme, $X$}

This scheme proposed in [20] is based on Ericsson's proposal [21-23] of static frequency reuse scheme and Siemen's proposal of semi-static ICIC. In this scheme, a part of the sub-bands is utilized by cell-edge UEs with full power and the whole spectrum is used by cell-centre users with reduced power level of the sub-bands which are available at the cell edge. If the cell-edge area of a particular cell becomes heavily loaded, the cell can borrow the sub-bands deployed in cell-edge area of adjacent cells. The scheme considers the variation of traffic load between cell-centre UEs and cell-edge UE as well as the traffic load changes among adjacent cells.

An example of X scheme is shown in Fig. 10. The cell-edge of cell 1 has heavy traffic load whereas cell-edge traffic load is common in cell 2, 4 and 6 and low in cell 3,5 and 7. So, the frequency sub-bands will be borrowed from cell edge area of cell 3, 5 and 7 by cell 1 . It is noted that, the frequency resource can only be borrowed when the traffic load in all the celledge area of cell 3,5 and 7 is low so that the cell-edge UEs in adjacent cells can not interfere with each other. 

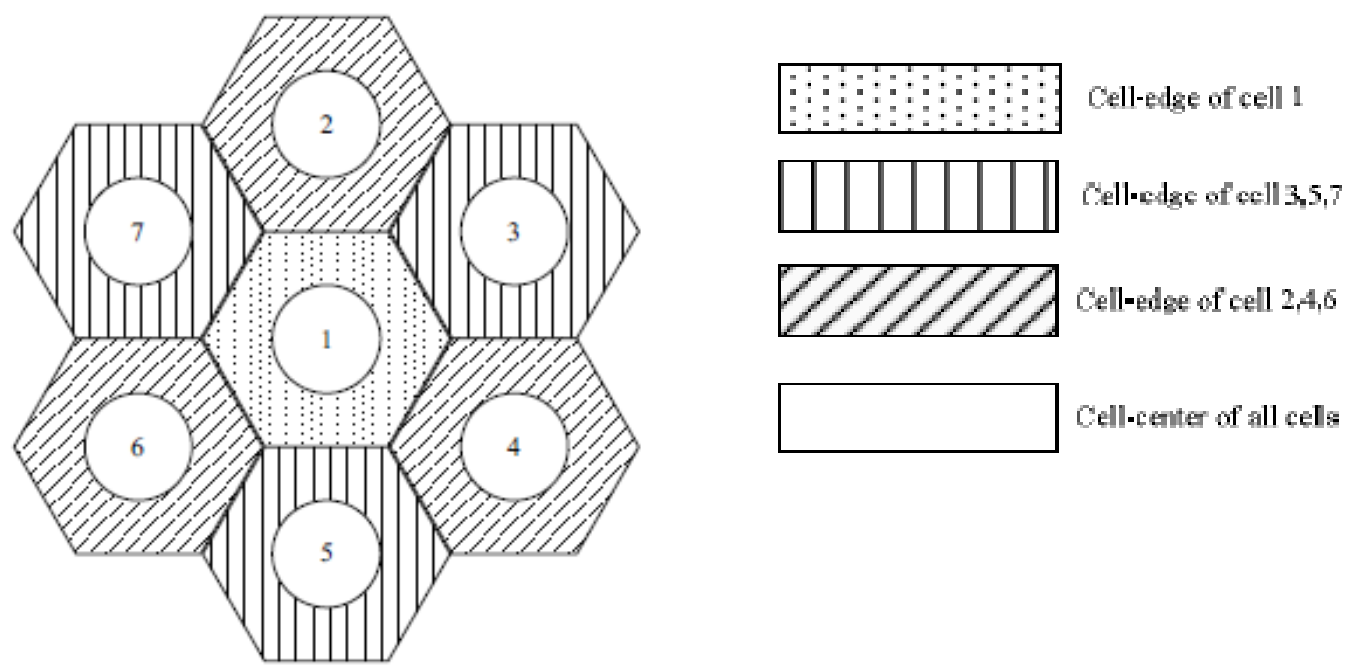

Cell-edge of cell $2,4,6$

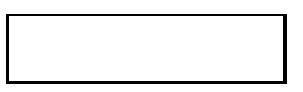

Cell-center of all cels

Fig. 9: Frequency reuse scheme of Siemen's proposal [20]
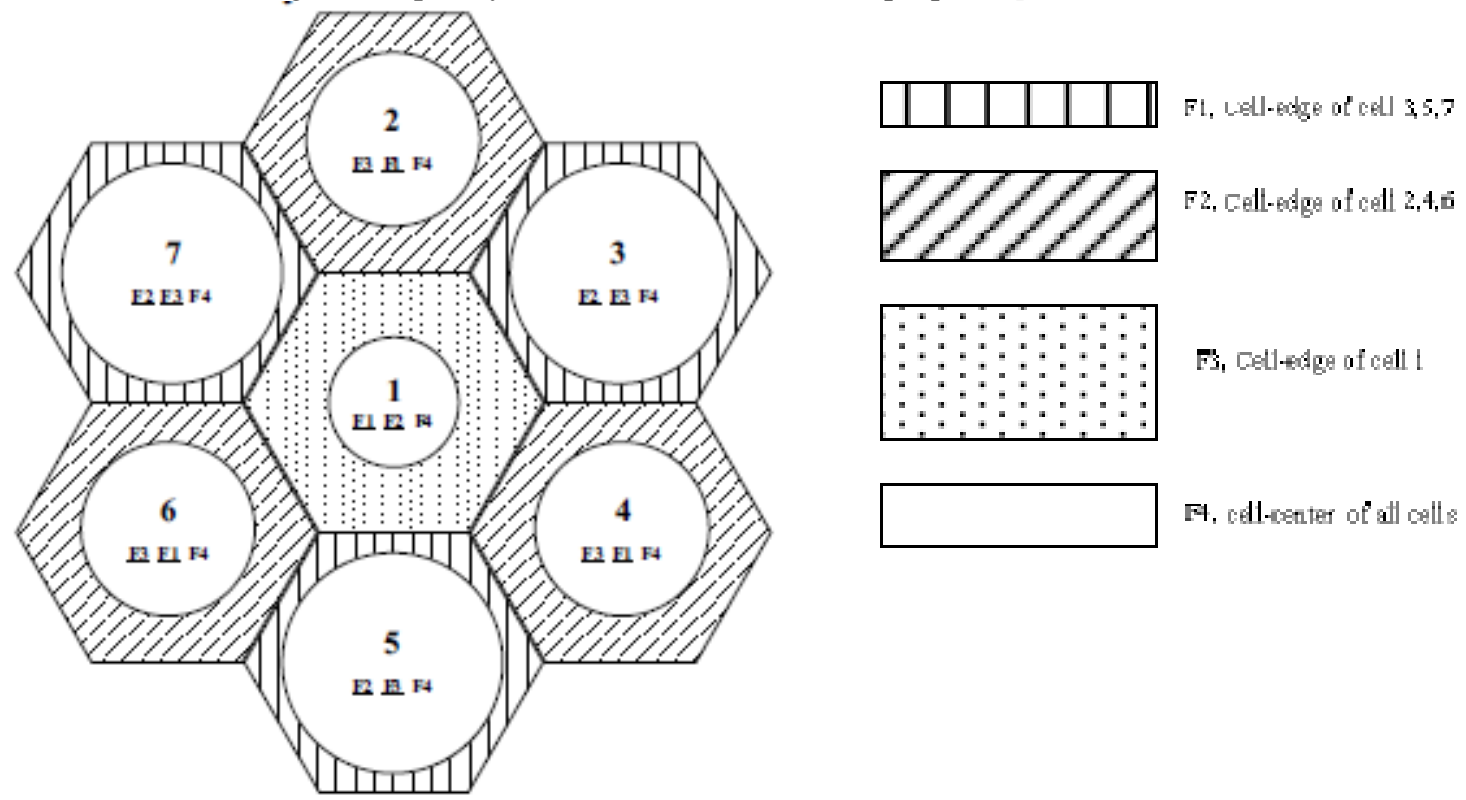

F2, Cell-edge of cell $2,4,5$

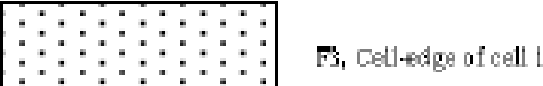

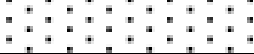

F4, cell-center of att calls

Fig. 10: Frequency reuse scheme of the proposal in [20]

\subsection{Dynamic Schemes}

The scale and complexity of modern cellular system have imposed the necessity to explore the cell coordinated based schemes as potential models for managing such highly complex system. The objectives of the proposed coordinated schemes mainly focus to maximize overall system throughput, minimize interference and/or minimize power usage [6]. Coordination based scheme can be classified as centralized, coordinated-distributed, semi-distributed and autonomous-distributed scheme.

\subsubsection{Centralized Scheme}

In this scheme, coordination is maintained by a centralized controller that collects all the channel state information (CSI) of each user existing in the system and assigns the available 
resource blocks to each eNB in such a way that capacity is maximized. Thus, each eNB need to forward the channel state information received from each UE to the central control unit and receive back the resource allocation information. But, it is hard task to do centralized scheduling because of the stringent time needed to exchange scheduling information and the large feedback information required by the users to transmit all the CSIs. That's why, LTE-A system has abolished the central control unit and relied on coordination among eNBs over the X2 interface without any centralized coordination in a flat architecture [4]. Some examples of this type of coordination based scheme can be found in [24-26].

\subsubsection{Semi-Distributed Scheme}

In semi-distributed schemes, coordination is usually implemented at two levels such as the central controlling entity level and the eNBs level. Like centralized scheme, a central controlling entity is deployed that controls a number of eNBs. However, in semi-distributed approach, each super-frame of bulk resources are allocated to each eNB by the central entity whereas in the centralized scheme the central entity allocates the channels directly to each user on frame-basis. Hence, in semi-distributed scheme, each eNB is in charge to allocate channels on the frame level to the users that are served. As the resource allocation task is distributed between eNBs and central controlling entity, as a whole the computational load of the scheme can be reduced. Some examples of semi-distributed schemes can be found in [27-30]. The semi-distributed scheme can be employed for eICIC (enhanced Inter Cell Interference Coordination) in HetNets (Heterogeneous Network) [6].

\subsubsection{Coordinated-Distributed Scheme}

In centralized and semi-distributed approaches, all the interference information on every resource block need to be collected at the central control entity, and in reality, the volume of this information required from eNBs to the central controller can be excessively large [29]. For this reason, the rate of exchanging information between eNB and central entity must be minimized, which results in degraded overall system performance. In coordinated-distributed schemes, central entity doesn't need to perform the coordination and resources are allocated only at the eNB level. But, still coordination between eNBs is required for exchanging CSI reports to maintain global ICIC. For practical implementation, the coordinated-distributed scheme is more efficient because it has distinct pros over the semi-distributed approach such as minimizing time and signalling overhead due to the systematic communication between eNBs and central controller, lessening the complexity of network infrastructure as central controller is eliminated here. Some examples of coordinated-distributed schemes reported in the literature can be found in [31-35]. Fig. 11 shows an example of coordinated-distributed scheme. 

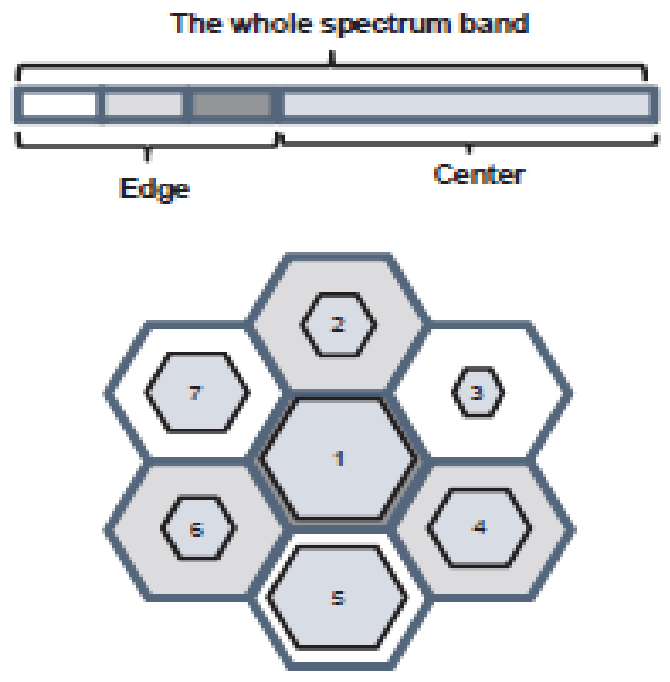

Fig. 11: Dynamic fractional frequency reuse scheme [35] - an example of coordinateddistributed scheme where cell centre boundaries are changed dynamically based on cell load, user behaviors, and interference situation from adjoin cells. As seen in figure, the cell centre areas are of different size in different cell and cell 1 has high load while cell 3 has lowest load. Consequently, the region of RF1 comes larger in cell 1 as compared with cell 3.

\subsubsection{Autonomous-Distributed Scheme}

In autonomous-distributed approaches, resource allocation is made only at the eNB level without any usage of central controlling entity for coordination. This is the similarity of autonomous-distributed schemes with coordinated-distributed schemes. On the other hand, dissimilar to coordinated-distributed schemes, coordination between eNBs is not required for autonomous-distributed schemes. For each eNB, channels are allocated by the eNB to its corresponding UEs depending on the local information gathered from its UEs. In autonomousdistributed scheme, it is possible to place the RBs anywhere (in a distributed-fashion) as required to enhance the system capacity, so the system acts as self-organizing system. In order to maintain network-wide ICIC and good fairness with autonomous-distributed schemes, some of the RBs of each eNB must be restricted by minimizing power level or not to use it at all which in turn reduces the ICI on those RBs for adjacent cells. Since, there is no coordination among eNBs in autonomous schemes, the RBs need to be restricted are selected on the basis of SINR values of those RBs. Low SINR level specifies that a resource block is being utilized by adjacent cells. When RBs are being restricted by $\mathrm{eNB}$, a scheme requires to make a compromise between the value of lessening the ICI in adjacent cells and the cost of utilizing the spectrum available [7]. Some example of autonomous-distributed ICIC schemes can be found in [36-40].

\section{INTERFERENCE RANDOMIZATION}

One of three ICI mitigation techniques is named as interference randomization. In interference randomization policy, the users' data are spread up over a distributed set of subcarriers so that interference scenario can be randomized and frequency diversity gain can be achieved. In interference randomization, in each cell the users' data are sequentially allocated over a timefrequency chunks. When all the requested transmission are allocated, subcarriers permutation is made in random manner so that each UE's transmission is arbitrarily spread up over the total time-frequency grid. Fig. 12 shows the allocation of subcarriers in a given cell before and after 
the pseudorandom permutation. In each interfering cell, the pseudorandom permutation is performed independently. The cell specific scrambling causes the interference spread up along with the transmission of a given user. As the coding is performed at the transmitter during transmission, the whole bit stream can be easily recovered at the receiving end. As interference system requires no signalling overhead for coordination among cells and less complex resource management, it is suitable for practical system [41].
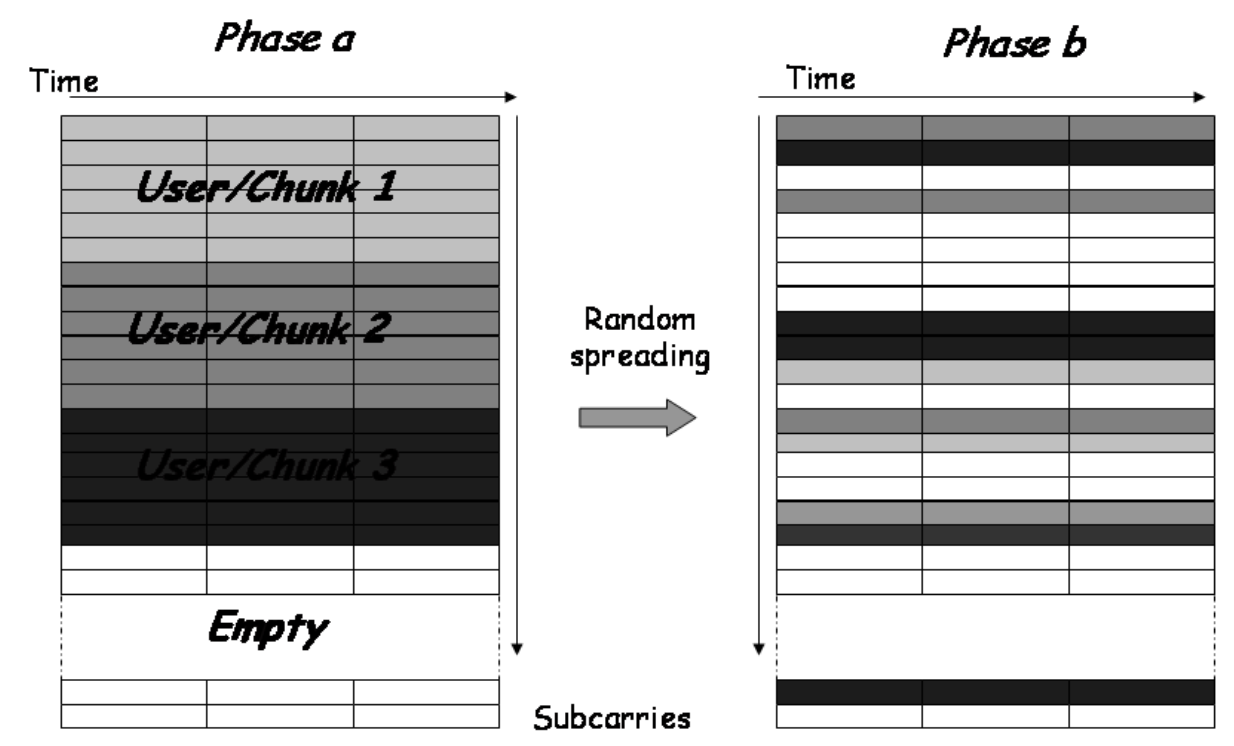

Fig. 12: Subcarrier allocation before (a) and after (b) random permutation [41]

\section{CONCLuSion}

Inter-cell interference coordination strategies can be considered as a scheduling scheme that takes into account the situation of adjacent cells to reduce the impact of inter-cell interference (IC) and improve the cell edge throughput. As a whole, the ICI avoidance schemes put restrictions of using downlink resources such as time/frequency/transmit power. The coordination of these restrictions helps to limit the generation of interference in a cellular network. Therefore, at the receiving end within the network coverage, SINR can be improved, which in turn provides an opportunity for getting increased data rate over the network coverage area. In this paper, a survey of interference avoidance schemes employed to mitigate the inter-cell interference problem occurred in downlink LTE system are studied. This paper presents a review of three main types of interference avoidance schemes, namely, static, semistatic and dynamic schemes. Also the basic concept of interference randomization is discussed. Our future work includes to review the existing interference cancellation schemes for LTE network as well as to propose an interference mitigation scheme for downlink LTE system. 
International Journal of Wireless \& Mobile Networks (IJWMN) Vol. 7, No. 1, February 2015

\section{REFERENCES}

[1] N. Himayat, S. Talwar, A. Rao and R. Soni, "Interference Management for 4G Cellular Standards," IEEE Communications Magazine, August 2010, Vol. 48(8), pp.86-92.

[2] A. Daeinabi, K. Sandrasegaran and X. Zhu, "Survey of Intercell Interference Mitigation Techniques in LTE Downlink Networks," Australasian Telecommunication Networks and Applications Conference, Nov. 2012, pp.1-6.

[3] E. Dahlman, S. Parkvall, J. Sköld, and P. Beming, 3G Evolution: HSPA and LTE for Mobile Broadband, Amsterdam; Oxford : Elsevier Academic Press, 2007.

[4] S. Sesia, I. Toufik, and M. Baker, LTE- The UMTS Long Term Evolution: From Theory to Practice, Chichester, U.K. Wiley, 2009.

[5] G. Boudreau, J. Panicker, N. Guo, R. Chang, N. Wang, and S. Virzic, "Interference Coordination and Cancellation for 4G Networks," IEEE Communications Magazine, Vol.47(4), pp.74-81, April 2009.

[6] A.S. Hamza, S.S. Khalifa, H. S. Hamza, and K. Elsayed, " A Survey on Inter-Cell Interference Coordination Techniques in OFDMA-Based Cellular Networks," IEEE Communications Surveys \& Tutorials, Fourth Quarter 2013, Vol.15(4), pp.1642-1670.

[7] G. Fodor, C. Koutsimanis, A. Rácz, N. Reider, A. Simonsson, and W. Müller, "Intercell Interference Coordination in OFDMA Networks and in the 3GPP Long Term Evolution System," Journal of Communications, 2009, Vol.4(7), p.445.

[8] Xuehong Mao, A. Maaref and Koon Hoo Teo, "Adaptive Soft Frequency Reuse for Inter-cell Interference Coordination in SC-FDMA based 3GPP LTE Uplinks," IEEE GLOBECOM 2008 2008 IEEE Global Telecommunications Conference, Nov. Dec. 2008, pp.1-6.

[9] F. Afroz, "Research in 4G Mobile Network," MES Project, University of Technology, May 2014.

[10] N. Saquib, E. Hossain, and D. Kim "Fractional Frequency Reuse for Interference Management in LTE-Advanced HetNets," IEEE Wireless Communications, Vol.20(2), pp.113-122, April 2013.

[11] S. Hussain, "Dynamic Radio Resource Management in 3GPP LTE," Blekinge Institute of Technology, Jan. 2009.

[12] R. Kwan and C. Leung, "A Survey of Scheduling and Interference Mitigation in LTE," Electrical and Computer Engineering, vol. 2010, 2010.

[13] F. Khan, "LTE for 4G Mobile Broadband: Air Interface Technologies and Performance," Cambridge University Press, 2009.

[14] 3GPP R1-050841, "Further Analysis of Soft Frequency Reuse Scheme," Huawei, 2005. Available: http://www.3gpp.org/ftp/tsg_ran/WG1_RL1/TSGR1_42/Docs/R1-050841.zip

[15] 3GPP R1-050507, "Soft Frequency Reuse Scheme for UTRAN LTE," Huawei, 2005. Available: http://www.3gpp.org/ftp/tsg_ran/WG1_RL1/TSGR1_41/Docs/R1-050507.zip

[16] IEEE C802.16m-08/782, "Fractional Frequency Reuse in Uplink," LG Electronics, 2008. Available: www.ieee802.org/16/tgm/contrib/C80216m-08_782.doc

[17] X. Zheng, B. Walke, "Resource Allocation and Reuse for Inter-Cell Interference Mitigation in OFDMA based Communication Networks," Wireless Internet Conference, March 2010, pp.1-6.

[18] R1-050738, Siemens, "Interference mitigation - Considerations and Results on Frequency Reuse", RAN WG1\#42, London, UK, Aug./Sept. 2005.

[19] R1-051366, "Aspects of Interference Mitigation by Coordination," Siemens, TSG-RAN WG1 Meeting \#43, Seoul, Korea, Nov. 2005.

[20] X. Fan, Chen Si and X. Zhang, "An Inter-Cell Interference Coordination Technique Based on Users' Ratio and Multi-Level Frequency Allocations," International Conference on Wireless Communications, Networking and Mobile Computing, September 2007, pp. 788-802.

[21] Ericsson, "Muting - further discussion and results," Tech. Rep. TSG-RAN WG1 R1-050763, 3rd Generation Partnership Project (3GPP), August-September 2005.

[22] Ericsson, "Inter-cell interference handling for E-UTRA," Tech. Rep. TSG-RAN WG1 R1-050764, 3rd Generation Partnership Project (3GPP), August-September 2005.

[23] Ericsson, "Downlink inter-cell interference coordination/avoidance evaluation of frequency reuse,” Tech. Rep. TSG-RAN WG1 R1-061374, 3rd Generation Partnership Project (3GPP), May 8-12, 2006.

[24] A. Gjendemsjo, D. Gesbert, G. E. Oien, and S. G. Kiani, OOptimal Power Allocation and Scheduling for Two-Cell Capacity Maximization," in Proc. 4th Int Modeling and Optimization in Mobile, Ad Hoc and Wireless Networks Symp., 2006, pp. 1-6. 
International Journal of Wireless \& Mobile Networks (IJWMN) Vol. 7, No. 1, February 2015

[25] S. Das, H. Viswanathan, and G. Rittenhouse, "Dynamic load balancing through coordinated scheduling in packet data systems," in Proc. IEEE INFOCOM 2003, 2003, vol. 1, pp. 786-796.

[26] T. Bonald, S. Borst, and A. Proutiere, "Inter-Cell Scheduling in Wireless Data Networks," in Proc. 11th European Wireless Conference 2005, 2005, pp. 1-7.

[27] M. Rahman and H. Yanikomeroglu, "Enhancing cell-edge performance: a downlink dynamic interference avoidance scheme with inter-cell coordination," IEEE Transactions on Wireless Communications, vol. 9, no. 4, pp.1414-1425, 2010.

[28] M. Rahman and H. Yanikomeroglu, "Multicell Downlink OFDM Subchannel Allocations Using Dynamic Inter-cell Coordination," in Proc. IEEE Global Telecommunications Conf. GLOBECOM '07, 2007, pp. 5220-5225.

[29] G. Li and H. Liu, "Downlink Radio Resource Allocation for Multi-Cell OFDMA System," IEEE Transactions on Wireless Communications, vol. 5, no. 12, pp. 3451-3459, 2006.

[30] M.C. Necker, "A Novel Algorithm for Distributed Dynamic Interference Coordination in Cellular Networks," in Proc. KiVS, pp. 233-238, 2011.

[31] A. Triki and L. Nuaymi, "Intercell Interference Coordination Algorithms in OFDMA Wireless Systems," in Proc. IEEE 73rd Vehicular Technology Conf. (VTC Spring), 2011, pp. 1-6.

[32] T. Q.S. Quek, Z. Lei, and S. Sun, "Adaptive interference coordination in multi-cell OFDMA systems," in IEEE 20th International Symposium on Personal, Indoor and Mobile Radio Communications, 2009, pp. 2380-2384.

[33] K. Dong, H. Tian, X. Li, and Q. Sun, “A Distributed Inter-Cell Interference Coordination Scheme in Downlink Multicell OFDMA Systems," in Proc. 7th IEEE Consumer Communications and Networking Conf. (CCNC), 2010, pp. 1-5.

[34] H. Kwon, W.-I. Lee, and B. Gi Lee, "Low-Overhead Resource Allocation with Load Balancing in Multi-cell OFDMA Systems," in IEEE 61st Vehicular Technology Conference, 2005, pp. 30633067.

[35] D. Kimura, Y. Harada, and H. Seki, "De-Centralized Dynamic ICIC Using X2 Interfaces for Downlink LTE Systems," in Proc. IEEE 73rd Vehicular Technology Conf. (VTC Spring), 2011, pp. $1-5$.

[36] A. L. Stolyar and H. Viswanathan, "Self-Organizing Dynamic Fractional Frequency Reuse in OFDMA Systems," in Proc. INFOCOM 2008. The 27th Conf. Computer Communications. IEEE, 2008, pp. 691-699.

[37] A. L. Stolyar and H. Viswanathan, "Self-Organizing Dynamic Fractional Frequency Reuse for Best-Effort Traffic through Distributed Inter-Cell Coordination," in Proc. IEEE INFOCOM 2009, 2009, pp. 1287-1295.

[38] S. Cicalo, V. Tralli, and A.I. Perez-Neira, "Centralized vs Distributed Resource Allocation in Multi-Cell OFDMA Systems," in IEEE 73rd Vehicular Technology Conference, 2011, pp. 1 - 6.

[39] S. Ko, H. Seo, H. Kwon, and B. G. Lee, "Distributed Power Allocation for Efficient Inter-cell Interference Management in Multi-cell OFDMA Systems," in 16th Asia-Pacific Conference on Communications, 2010, pp. 243-248.

[40] Q. D. La, Y. H. Chew, and B.-H. Soong, \An Interference Minimization Game Theoretic Subcarrier Allocation Algorithm for OFDMA-Based Distributed Systems," in IEEE Global Telecommunications Conference, 2009, pp. 1-6.

[41] R. Bosisio and U. Spagnolini, "Interference Coordination vs. Interference Randomization in Multicell 3GPP LTE System," IEEE Wireless Communications and Networking Conference, March April 2008, pp.824-829. 


\section{Authors}

Farhana Afroz obtained her MS degree and B.Sc. (Honours) degree from the department of Applied Physics, Electronics and Communication Engineering of University of Dhaka, Bangladesh. Her undergraduate and postgraduate-level research works were in material science and digital signal processing respectively. She is now pursuing Master of Engineering studies with Telecommunication

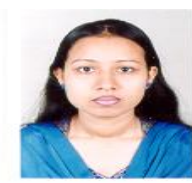
Engineering major in University of Technology, Sydney, Australia. She worked as a Lecturer for the department of Electronics and Telecommunication Engineering in University of Liberal Arts Bangladesh, during 2007-2010 time period. Her current research interest includes 4G mobile networks and digital signal processing.

Dr Kumbesan Sandrasegaran is an Associate Professor at UTS and Centre for RealTime Information Networks (CRIN). He holds a PhD in Electrical Engineering from McGill University (Canada) (1994), a Master of Science Degree in Telecommunication Engineering from Essex University (1988) and a Bachelor of Science (Honours) Degree in Electrical Engineering (First Class) (1985). His current research work focuses on two main areas (a) radio resource management in mobile networks, (b) engineering of remote monitoring systems for novel applications with industry through the use of embedded systems, sensors and communications systems. He has published over 100 refereed publications and 20 consultancy reports spanning telecommunication and computing systems.

Haider Al Kim got his B.Sc. in Information and Communication Engineering from AlKhwarizmi Engineering College, University of Baghdad, Baghdad, Iraq in 2008. He pursues his Master degree in Telecommunication Networks from University of Technology Sydney (UTS), Sydney, Australia in 2014 under the supervision A. Prof. Kumbesan Sandrasegaran. Working and research areas are Wireless Telecommunication, Mobile Network, Network Management, Network Design and Implementation and Data Analysis and Monitoring .He is senior network engineer with more than 5 years work experience in networks and telecommunication industry at University of Kufa, Iraq. He is also a Cisco Certificate holder (ID: CSCO11773718) and Cisco instructor at Al-Mansour College, Baghdad, Iraq in 2010-2011. 